\title{
On the uncertainty of height anomaly differences predicted by least-squares collocation
}

https://doi.org/10.1515/jogs-2020-0111

Received February 4, 2020; accepted May 17, 2020

\begin{abstract}
A network of pointwise available height anomalies, derived from levelling and GPS observations, can be densified by adjusting a gravimetric quasigeoid using least-squares collocation. The resulting type of Corrector Surface Model (CSM) is applied by Norwegian surveyors to convert ellipsoidal heights to normal heights expressed in the official height system NN2000. In this work, the uncertainty related to the use of a CSM to predict differences in height anomaly was sought. As previously, the application of variograms to determine the local statistical properties of the adopted collocation model led to predictions that were consistent with their computed uncertainties. For the purpose of predicting height anomaly differences, the effect of collocation was seen to be moderate in general for the small spatial separations considered $(<10 \mathrm{~km})$. However, the relative impact of collocation could be appreciable, and increasing with distance, near the network. At last, it was argued that conservative uncertainties of height anomaly differences may be obtained by rescaling output of a grid interpolation by $\sqrt{\Delta}$, where $\Delta$ is the spatial separation of the two locations for which the difference is sought.
\end{abstract}

Keywords: corrector surface model, height anomaly differences, uncertainty, variogram

\section{Introduction}

A problem encountered in geodesy is the consistent description of information provided by different observation techniques on a spatially variable quantity. Typically, one technique provides pointwise accessible information on the quantity in question, while a second data type yields more densely available, but presumed less precise information. An example of such a quantity can be the height

^Corresponding Author: E. Mysen: Geodetic Institute, Norwegian Mapping Authority, Norway, E-mail: eirik.mysen@kartverket.no anomaly, which is pointwise available from GPS ${ }^{1}$ observations and levelling, and densely available by sampling a gravimetric quasigeoid model (e.g. [25], [40], [36], [5]).

One approach to the combination of pointwise and densely available information on the height anomaly is to model the discrepancy between the two information types explicitly. Often, the model adopted for the discrepancy is composed of a simple deterministic trend and its misfit interpreted as correlated stochastic error. A consistent prediction of the height anomaly can then be made, using least-squares collocation (e.g. [22], [18], [31]), wherever the gravimetric quasigeoid model is available. These predictions define the Corrector Surface Model (CSM) (e.g. [16]). A CSM can be used by surveyors to convert ellipsoidal heights to normal heights expressed in an official height system.

The presented work will provide more context for, and elaborate on, a previous approach [23] to compute representative uncertainties in empirical least-squares collocation. More specifically, a trend in the form of a local bias will be allowed, and a weighting strategy in the determination of the stochastic error's characteristics will be implemented. However, the main purpose is to gain an understanding of how the uncertainties of height anomaly differences behave, when derived from a CSM. Previous studies that discuss the errors of computed height (anomaly) differences include [15], [32], [31], [6] and [1]. In the following, the effect of collocation is investigated more closely with the purpose of providing a representative conservative rule, using the data set that realizes the official Norwegian height system NN2000.

First, some details on the data types and least-squares collocation will be given, followed by a discussion of the applied variogram approach to the determination of stochastic properties. To check the adopted statistical models, their consistency with prediction errors will be studied. Finally, the uncertainty of predicted height anomaly differences will be mapped, and general properties sought.

1 Global Positioning System. 


\section{Least-squares collocation}

The Norwegian height system NN2000 is realized by 3,297 normal heights $\hat{H}_{i}^{\star}$, see Fig. 1, expressed in the zero-tide system (e.g. [4], [24]). The heights, obtained by levelling, are referenced to the year 2000.5 using the land uplift model NKG2005LU ([35], [39]) to account for the significant postglacial rebound across Fennoscandia. At each of these

\section{$200 \mathrm{~km}$}

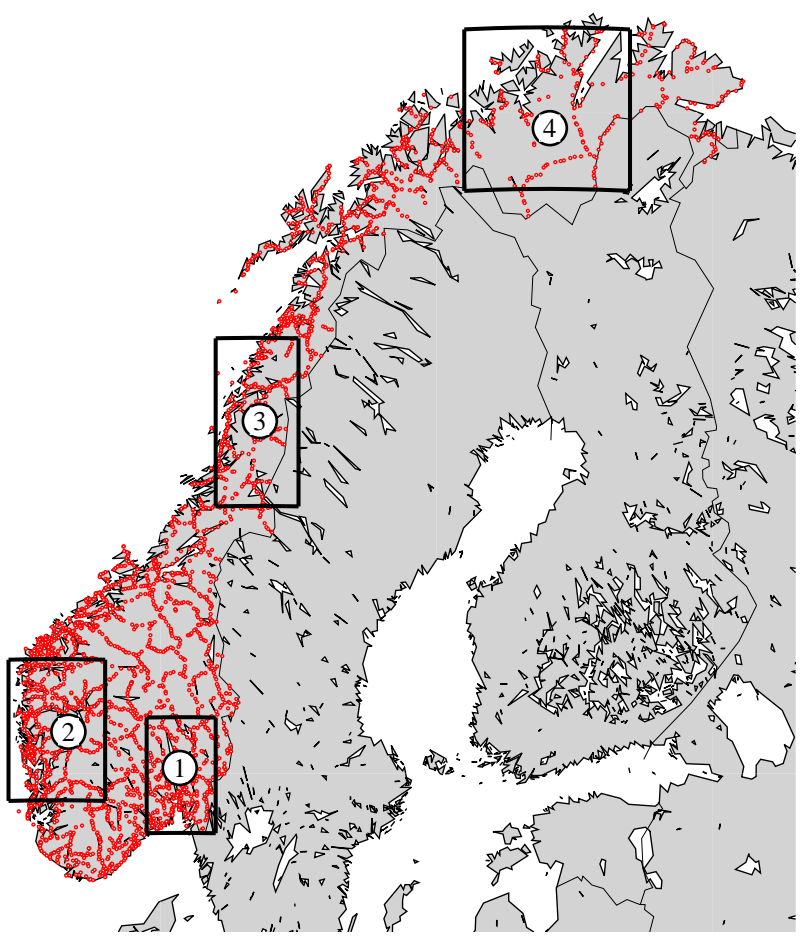

Figure 1: Mainland Norway is shown, together with the locations of the 3,297 normal heights (red dots) that realize the Norwegian height system NN2000. Statistical properties for the four marked locations are given in Table 1 . Results from four sample regions containing these locations are given in Table 3.

locations $i$, non-tidal (e.g. [28], [27]) ellipsoidal heights $\hat{h}_{i}$ expressed in the ETRS89 realization EUREF89 [14] have been derived from GPS observations, yielding pointwise available estimates $p_{i}$ of height anomalies $\zeta_{i}$

$$
p_{i}=\hat{h}_{i}-\hat{H}_{i}^{\star}=\zeta_{i}+n_{i} .
$$

Since the systematic errors of the pointwise available data type cannot be determined by the following method [23], they are formally set to zero. As the error of the normal heights that realize NN2000 is considered to be mostly systematic, the term $n_{i}$ of Eq. (1) can be identified as the spatially uncorrelated error of the ellipsoidal heights

$$
E\left(n_{i} n_{j}\right) \equiv W_{i j}=\omega_{1} \delta_{i j}
$$

Here, $E(\cdot)$ is the statistical expectancy, $\delta_{i j}$ is the Kronecker delta, and $\omega_{1}$ is a covariance parameter yet to be specified.

Equations (1) and (2) can be seen as a definition of the type of height anomaly involved. That is, the $\zeta_{i}$ of Eq. (1) deviate from true height anomalies due to systematic errors of the involved observation techniques, errors of the applied land uplift model, and different tidal systems of EUREF89 (non-tidal crust) and NN2000 (mean crust). However, the latter inconsistency is by design so that, when the Corrector Surface Model is subtracted, ellipsoidal heights expressed in EUREF89 are transformed to normal heights in NN2000.

Height anomaly estimates $d_{i}$ are also available from quasigeoid surface models based on gravimetric measurements. In this work, the NMA2013v30 quasigeoid model computed by colleague O. Omang using GRAVSOFT [34], and the NKG2015 quasigeoid model [41] developed under the umbrella of the Nordic Geodetic Comission (NKG), are considered. Since gravimetric quasigeoids are continuous models ${ }^{2}$ of a continuous surface, their deviation from the true height anomalies $\zeta_{i}$ are systematic

$$
\begin{aligned}
d_{i} & =(\text { NMA2013v30/NKG2015) } \\
& =\zeta_{i}-a_{i} x-s_{i}
\end{aligned}
$$

where $a_{i} x$ is a deterministic component often called the trend, given by the constant parameter vector $x$. The random and spatially correlated $s_{i}$ of Eq. (5) is often referred to as the signal [22, p. 76].

The NMA2013v30 and NKG2015 quasigeoid models do not correspond precisely to the height anomalies defined by Eqs. (1) and (2), partly because the former are non-tidal (colleague Ove Omang, personal communication). The latter inconsistency is, among other deterministic discrepancies, accounted for by the estimation of a local bias (trend) using the scheme described in the following.

One can define a new observation $z_{i}$ as the discrepancy between the two fundamental data types

$$
z_{i} \equiv p_{i}-d_{i}=a_{i} x+\varepsilon_{i},
$$

where the stochastic process $\varepsilon_{i}$ is given by

$$
\varepsilon_{i}=s_{i}+n_{i}
$$

2 Bilinear interpolation of a grid. 
with covariance

$$
R_{i j} \equiv E\left(\varepsilon_{i} \varepsilon_{j}\right)=W_{i j}+C_{i j}, \quad C_{i j} \equiv E\left(s_{i} s_{j}\right) .
$$

Now, assume that we can compute a prediction $\tilde{s}_{k}$ of $s_{k}$ at a location $k$ where only the dense data type $d_{k}$ is available, based on the observations $z_{i}$ of Eq. (4). Likewise, let $\hat{x}$ be an estimate of $x$ determined by the same observations. We can then provide predictions $\tilde{\zeta}_{k}$ of the height anomaly by adjusting the gravimetric quasigeoid according to

$$
\tilde{\zeta}_{k}=d_{k}+\tilde{z}_{k}, \quad \tilde{z}_{k}=a_{k} \hat{x}+\tilde{s}_{k},
$$

noting that the prediction $\tilde{n}_{k}$ of a spatially uncorrelated $n_{k}$ is zero.

The observations of Eq. (4) may be written on matrix form

$$
z=A x+\varepsilon, \quad \varepsilon=s+n,
$$

where the rows of $A$ are given by $a_{i}$ of Eq. (4). A leastsquares prediction $\tilde{s}_{k}$ of the signal at location $k$ is then [22]

$$
\tilde{s}_{k}=c_{k}^{T} R^{-1}(z-A \hat{x}),
$$

with $R=E\left(\varepsilon \varepsilon^{T}\right)$ of Eq. (6), and the $i$ 'th component of the vector $c_{k}$ defined by

$$
c_{k, i}=E\left(s_{k} s_{i}\right),
$$

where the index $i$ here refers to a location where we have an observation $z_{i}$. Above, $T$ is the matrix transposition operator.

The least-squares estimate $\hat{x}$ of the trend vector $x$ derived from Eq. (8) is given by

$$
\hat{x}=\left(A^{T} R^{-1} A\right)^{-1} A^{T} R^{-1} z .
$$

From a statistical perspective, Eqs. (9) and (11) yield the best linear unbiased prediction (BLUP) (e.g. [9], [12]) in the form of Eq. (7). These height anomaly predictions define the Corrector Surface Model (CSM). An estimate of a location's normal height in NN2000 can then be obtained by subtracting the sampled CSM from a location's ellipsoidal height expressed in the reference frame EUREF89.

The error of $\tilde{\zeta}_{k}$ can be obtained with the use of Eqs. (4), (5) and (7)

$$
\tilde{\zeta}_{k}=p_{k}+\tilde{z}_{k}-z_{k}=\zeta_{k}+\tilde{z}_{k}-\left(z_{k}-n_{k}\right)+n_{k},
$$

which yields

$$
\tilde{\zeta}_{k}-\zeta_{k}=a_{k} \delta \hat{x}+\delta \tilde{s}_{k}+n_{k}
$$

where $\delta \hat{x} \equiv \hat{x}-x$ and $\delta \tilde{s}_{k} \equiv \tilde{s}_{k}-s_{k}$. The error's variance is then derivable from Eq. (13) using appropriate a posteriori error covariances

$$
E\left(\delta \hat{x} \delta \hat{x}^{T}\right), \quad E\left(\delta \tilde{s}_{k}^{2}\right), \quad E\left(\delta \hat{x} \delta \tilde{s}_{k}\right),
$$

given by [22, p. 128].

Later, we will have use of an expression for the residual

$$
e \equiv z-A \hat{x}=P z=P \varepsilon,
$$

where $P$ is the idempotent matrix

$$
P=I-A\left(A^{T} R^{-1} A\right)^{-1} A^{T} R^{-1}, \quad P^{2}=P,
$$

with the properties

$$
R^{-1} P=P^{T} R^{-1} .
$$

The latter matrix is positive semidefinite. To see this, note that $R$ as a covariance matrix must be chosen to be [17, $\mathrm{p}$. $100]$ symmetric and positive semidefinite. If we restrict out attention to the strictly positive definite case, the inverse $R^{-1}$ exists and has a square-root $R^{-1 / 2}[19, \mathrm{p} .16]$ so that

$$
R^{-1} P=R^{-1 / 2} \Omega R^{-1 / 2} .
$$

As a symmetric matrix, $\Omega$ can be diagonalized (ibid.) $\Omega=$ $\Gamma \Pi \Gamma^{T}$, where the entries of the diagonal $\Pi$ are either 0 or 1 since $\Omega$ also is idempotent [17, p. 49]. We then obtain

$$
b^{T} R^{-1} P b=\left(\Gamma R^{-1 / 2} b\right)^{T} \Pi\left(\Gamma R^{-1 / 2} b\right) \geq 0,
$$

for all vectors $b$.

\section{Modelling}

To obtain the uncertainty $\delta \Delta \tilde{\zeta}_{k l}$ of predicted height anomaly differences

$$
\Delta \tilde{\zeta}_{k l}=\tilde{\zeta}_{k}-\tilde{\zeta}_{l},
$$

we must find a suitable observation model. As in [23], a model on the from of Eq. (8) will be determined at each location from nearby observations. The corresponding moving window approach is not rigorous, but can be sufficient for predictions over the relatively small spatial separations involved. Since the validity of each model is restricted to a limited geographic region, simple biases will be adopted as local trends $A x$ of Eq. (8).

In this study, the a priori covariance $R$ of Eq. (6) at a location will be based on differences $z_{i}-z_{j}$ of surrounding observation pairs $z_{i}$ and $z_{j}$. One advantage with this variogram approach, closely related to the statistic introduced by [7] and extensively applied in spatial statistics since [21], is that it will not depend on a trend that consists of a constant only. This property can be particularly important if computed (formal) uncertainties are to be representative. For instance, the error of the estimate of the trend 
using Eq. (11) can be large if the signal $s$ in the region of focus is large and correlated. Methods based on the assumption that the residual $e$ of Eq. (15) is a good approximation of the effective noise $\varepsilon$ of Eq. (8) can therefore lead to [3, p. 71] biased estimates of $R$.

In this respect, the use of variograms to compute the covariance does not have an advantage in comparison to methods based on error contrasts (e.g. [11]), like LS-VCE [33] and REML ([26], [10]). However, in their most basic forms, the latter methods are dependent on a choice of $R$ that is representative for all data points, and therefore all spatial separations, whereas this is not necessary using the variogram.

To be more specific, the local statistics is determined by the $m=150$ observations $z_{i}$ closest to the location of interest. This particular number represents a balance between two conflicting properties of the empirical covariance: one wants to have enough observations to make the structure of $R$ discernible, but not so many that the matrix no longer describes local conditions. We can then form $m(m-1) / 2$ unique differences $z_{i}-z_{j}$, and organize these in distance classes according to the observations' spatial separation $\Delta_{i j}$. That is, with the parametrization solely by $\Delta_{i j}$, the resulting measure of statistical properties is averaged with respect to direction. In the same way as the covariogram is calculated from the average of the product $e_{i} e_{j}$ of residuals in each distance class, the classical variogram $2 \hat{\gamma}(\Delta)$ is obtained by the class average of $\left(z_{i}-z_{j}\right)^{2}$ (e.g. [2]).

Based on observed variograms for mainland Norway, see [23], the positive definite ([30], [13]) form

$$
C=E\left(s_{i} s_{j}\right)=\omega_{2} \exp \left(-\Delta_{i j} / \omega_{3}\right),
$$

is chosen. As a result, the expectancy $2 \gamma(\Delta) \equiv E(2 \hat{\gamma})$ of the empirical variogram is given by

$$
\begin{aligned}
E(2 \hat{\gamma}) & =2 E\left(n_{i}^{2}\right)+2 E\left(s_{i}^{2}\right)-2 E\left(s_{i} s_{j}\right) \\
& =2 \omega_{1}+2 \omega_{2}\left[1-\exp \left(-\Delta / \omega_{3}\right)\right]
\end{aligned}
$$

for spatial separations $\Delta \neq 0$.

Now, an approximation of the dispersion of $2 \hat{\gamma}$ is given by [2]

$$
E\left[(2 \hat{\gamma}-2 \gamma)^{2}\right] \approx 2(2 \gamma)^{2} / N_{\Delta} \equiv \mathcal{W}^{-1},
$$

where $N_{\Delta}$ is the number of samples in the distance class defined by $\Delta$, typically peaking for intermediary spatial separations. A commonly applied (see [8], [20]) weighted least-squares estimation scheme can then be formulated for the determination of the covariance parameters $\omega_{i}$. That is, with the empirical variogram $2 \hat{\gamma}(\Delta)$ as observations, and the variogram $2 \gamma$ itself as the model, covariance parameter estimates can be obtained by the minimization of

$$
\sum_{\Delta}(2 \hat{\gamma}-2 \gamma) \mathcal{W}(2 \hat{\gamma}-2 \gamma)
$$

with respect to $\omega_{i}$, where the weights $\mathcal{W}(\Delta)$ are given by Eq. (23). In this work, the minimization of this non-linear function is accomplished using the downhill simplex algorithm [29, p. 402] as applied by [38].

Solving Eq. (24) with observations based on the NMA2013v30 quasigeoid model, the estimates $\hat{\omega}_{i}$ of $\omega_{i}$ given in Table 1 are obtained for the four marked locations of Fig. 1. Clearly, the signal statistics is inhomogeneous. To obtain these results, the model is fitted to the empirical variogram only up to half the maximum available distance, which is advantageous for several reasons. This is

Table 1: The covariance parameters $\omega_{i}$ of Eq. (22) are estimated at the specified locations of Fig. 1 using the 150 closest observations.

\begin{tabular}{cccc}
\hline location & $\hat{\omega}_{1}\left[\mathrm{~cm}^{2}\right]$ & $\hat{\omega}_{2}\left[\mathrm{~cm}^{2}\right]$ & $\hat{\omega}_{3}[\mathrm{~km}]$ \\
\hline \hline 1 & $\equiv 0.5$ & 12 & 84 \\
\hline 2 & $\equiv 0.5$ & 21 & 7.4 \\
\hline 3 & $\equiv 0.5$ & 24 & 63 \\
\hline 4 & $\equiv 0.5$ & 19 & 41 \\
\hline
\end{tabular}

done partly since the reduction of the a priori covariance to the form of Eq. (21) can be unsubstantiated for large spatial separations. Also, the impact of a possible unmodelled linear trend on the determination of the covariance properties is reduced. That the validity of the model is limited to shorter distances is usually not a problem [3, p. 168] since predictions are mostly dependent on nearby observations.

As can be seen from Table 1 , the noise variance $\hat{\omega}_{1}$ has been defined since its estimation can be unstable in certain regions. The adopted value is at terms with the expected precision for the non-tidal ellipsoidal heights, believed to be between 5 and $10 \mathrm{~mm}^{3}$ based on repeated measurements (colleagues Torbjørn Nørbech and Olav Vestøl, personal communication), as well as the asymptotic properties of the empirical variograms of this work. Since $\hat{\omega}_{2}$ is in general considerably larger than $\hat{\omega}_{1}$, a reasonable definition of $\hat{\omega}_{1}$ does not alter the estimates $\hat{\omega}_{2}$ and $\hat{\omega}_{3}$ significantly.

As an example, the variogram for the Hordaland location (2) of Fig. 1 is shown as Fig. 2. The correlation length $\hat{\omega}_{3}$ is known to be very short in this region, but the signal magnitude $\hat{\omega}_{2}$ is quite average. It should be noted that, based on simulations, the wavy form of the empirical var-

3 One standard deviation. 


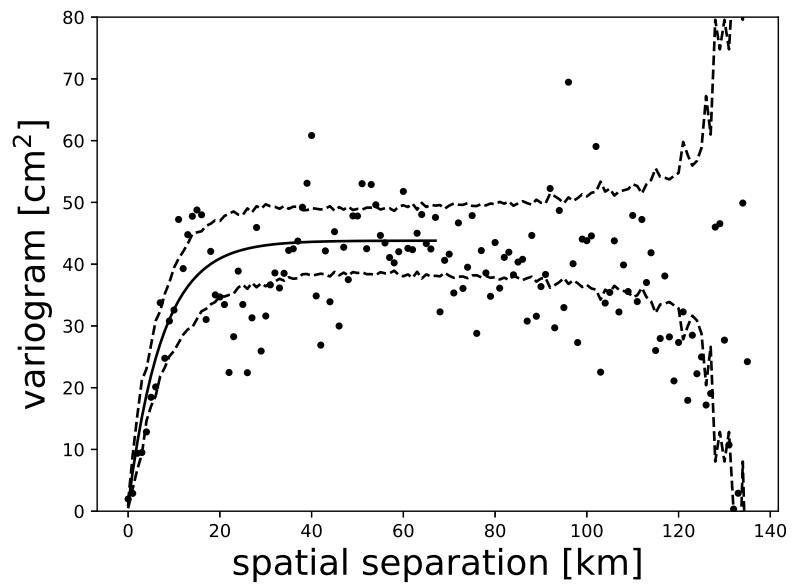

Figure 2: The empirical variogram (NMA2013v30) is shown for the Hordaland location (2) of Fig. 1, together with a fit (solid line). The variogram's expected dispersion is marked by dashed lines.

iogram seen in Fig. 2 can be a result of observation geometry, and therefore not necessarily a manifestation of the actual variogram. In Fig. 2 the limits defined by Eq. (23) are shown, fairly at terms with the observed dispersion.

\section{Consistency}

There are many ways to test a model, each approach having a specific sensitivity to certain observation geometries and target parameters. As in [23], a type of dynamic leave$n$-observations-out cross validation will be considered in the following, which corresponds to how least-squares collocation is applied in densification.

Assume that we disregard those observations that are within a distance $\Delta_{\min }$ of a location $i$. A prediction $\tilde{z}_{i}$ of the observation $z_{i}$ at location $i$ can then be made according to Eq. (7), based on the 20 closest observations outside the circle defined by the radius $\Delta_{\min }$. This particular number stems from the estimates' convergence properties. That is, significant changes are not observed by increasing the number further. The signal covariance is still determined from the 150 observations nearest to the location, but now with the additional condition that they must also be located outside the radius $\Delta_{\min }$, leading to the prediction error

$$
\tilde{\delta}_{i} \equiv z_{i}-\tilde{z}_{i}=p_{i}-\tilde{\zeta}_{i}
$$

That is, only observations $z_{j}$ more than $\Delta_{\min }$ away from $z_{i}$ are used to derive the local statistics and compute the prediction $\tilde{z}_{i}$.
These predictions can then be normalized by their formal error $\tilde{\sigma}_{i}$ derivable from the expressions provided by [22], as mentioned previously. The Root-Mean-Square (RMS)' of $\tau_{i}$, where $\tau_{i}$ is given by

$$
\tau_{i} \equiv \frac{\tilde{\delta}_{i}}{\tilde{\sigma}_{i}}
$$

can be calculated. Since the prediction errors are normalized by the predictions' formal error, this approach does not necessarily measure optimality, but consistency.

Table 2 shows that the formal error seems consistent with the prediction error on a national scale. A Root-Mean-

Table 2: Root-Mean-Squares (RMS) of $\tau_{i}$ are shown (NMA2013v30). All observations within a distance $\Delta_{\min }$ of location $i$ are disregarded in the computation of the prediction at location $i$. The sample consists of the $m=3,297$ observations available for mainland Norway.

\begin{tabular}{cccc}
\hline$\Delta_{\min }[\mathrm{km}]$ & $\mathrm{RMS}$ & $\tau_{\min }$ & $\tau_{\max }$ \\
\hline \hline 5 & 1.07 & -5.7 & 4.5 \\
\hline 10 & 1.04 & -5.3 & 3.9 \\
\hline 20 & 0.96 & -5.5 & 3.2 \\
\hline
\end{tabular}

Square of $\tau_{i}$ close to one has also been established for a number of smaller regions, see Table 3. Included as Fig. 3 is the distribution of $\tau_{i}$ with $\Delta_{\min }=10 \mathrm{~km}$ for the 539 observations of the Hordaland set, roughly following the normal distribution. This similarity is not always so clear for other areas, which can be expected due to correlations between prediction errors at adjacent locations.

Table 3: Root-Mean-Squares (RMS) of $\tau_{i}$ are shown below for the marked regions of Fig. 1 (NMA2013v30). Observations closer than $\Delta_{\min }=10 \mathrm{~km}$ to each prediction point are not used to make the prediction (or determine the local statistics) at that point.

\begin{tabular}{ccccc}
\hline region & RMS & $\tau_{\min }$ & $\tau_{\max }$ & samples $(m)$ \\
\hline \hline Oslo (1) & 1.11 & -4.5 & 3.9 & 493 \\
\hline Hordaland (2) & 1.10 & -3.6 & 3.5 & 539 \\
\hline Nordland (3) & 1.21 & -4.5 & 2.6 & 231 \\
\hline Finnmark (4) & 0.89 & -2.6 & 2.2 & 141 \\
\hline
\end{tabular}

\section{Height anomaly differences}

If the adopted model parameters are representative, we can obtain a picture of how the uncertainty of predicted 


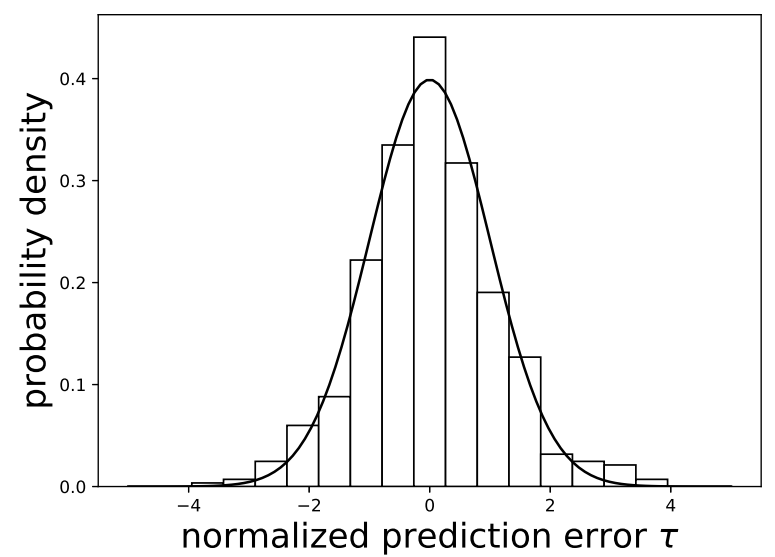

Figure 3: The distribution of normalized prediction errors for the Hordaland region (NMA2013v30). Observations closer to the prediction point than $\Delta_{\min }=10 \mathrm{~km}$ are excluded from the prediction algorithm.

height anomaly differences behaves. For this purpose, note that

$$
\begin{aligned}
\tilde{\zeta}_{k}-\tilde{\zeta}_{l}= & \left(\zeta_{k}-\zeta_{l}\right)+\left(a_{k}-a_{l}\right) \delta \hat{x} \\
& +\delta \tilde{s}_{k}-\delta \tilde{s}_{l},
\end{aligned}
$$

from Eq. (13). Since the trend consists of a local bias, we have $a_{k}-a_{l}=0$. As a result, we obtain

$$
\begin{aligned}
\left(\delta \Delta \tilde{\zeta}_{k l}\right)^{2}= & E\left[\left(s_{k}-s_{l}\right)^{2}\right]+E\left[\left(\tilde{s}_{k}-\tilde{s}_{l}\right)^{2}\right] \\
& -2 E\left[\left(\tilde{s}_{k}-\tilde{s}_{l}\right)\left(s_{k}-s_{l}\right)\right]
\end{aligned}
$$

where

$$
\left(\delta \Delta \tilde{\zeta}_{k l}\right)^{2} \equiv E\left\{\left[\tilde{\zeta}_{k}-\tilde{\zeta}_{l}-\left(\zeta_{k}-\zeta_{l}\right)\right]^{2}\right\} .
$$

The second term on the right hand side of Eq. (28) is

$$
\begin{aligned}
E\left[\left(\tilde{s}_{k}-\tilde{s}_{l}\right)^{2}\right] & =\Delta c_{k l}^{T} R^{-1} P E\left(\varepsilon \varepsilon^{T}\right) P^{T} R^{-1} \Delta c_{k l} \\
& =\Delta c_{k l}^{T} R^{-1} P \Delta c_{k l},
\end{aligned}
$$

with the use of Eqs. (15), (16) and (17), where $c_{k}$ is defined by Eq. (10) and

$$
\Delta c_{k l}=c_{k}-c_{l} .
$$

To proceed, we note that

$$
E\left(\varepsilon s_{k}\right)=c_{k},
$$

which yields

$$
\left(\delta \Delta \tilde{\zeta}_{k l}\right)^{2}=E\left[\left(s_{k}-s_{l}\right)^{2}\right]-\Delta c_{k l}^{T} R^{-1} P \Delta c_{k l} .
$$

The first term on the right hand side of Eq. (33) represents the uncertainty related to the direct use of gravimetric quasigeoid to compute height anomaly differences, while the second term quantifies the collocation effect. From Eq.
(19) we know that this latter term is negative (or zero), resulting in the natural conclusion that collocation reduces the uncertainty (or leaves it unchanged). Or, if we neglect the collocation term, we obtain a conservative estimate of the uncertainty related to predicted height anomaly differences, which has a simple analytic form derivable from Eq. (21).

Figure 4 shows the square root of the conservative first term of Eq. (33) for the NMA2013v30 quasigeoid model, using the separation $\Delta=1 \mathrm{~km}$. This region has been cho-
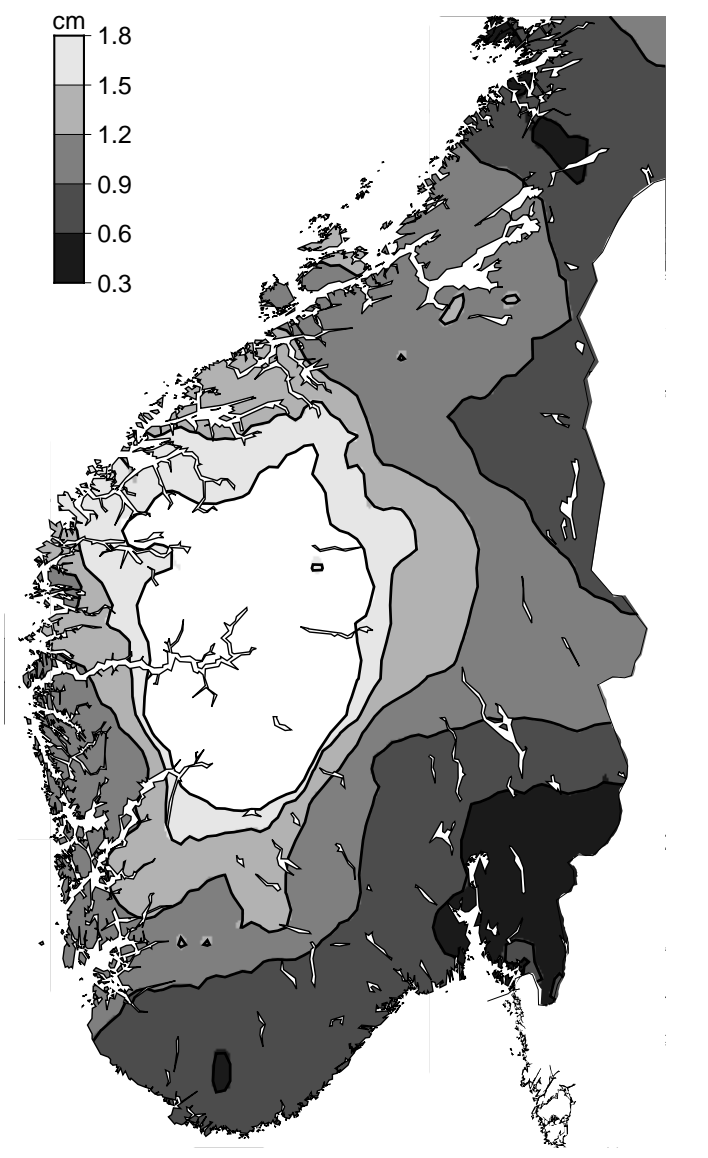

Figure 4: The uncertainty of height anomaly differences of the quasigeoid NMA2013v30 are shown, for locations separated by 1 $\mathrm{km}$. White represents off-scale values.

sen since it contains large contrasts in performance, with large uncertainties in the mountainous central and western parts. It can be noted that the variations seen in Fig. 4 are a direct expression of the signal statistics' inhomogeneity. Clearly, the variations in statistical properties can be substantial.

As for spatial separations $\Delta$ other than $1 \mathrm{~km}$, one can multiply the values of Fig. 4 by $\sqrt{\Delta[\mathrm{km}]}$, as long as $\Delta$ remains shorter than about $1 / 2$ of the correlation length $\hat{\omega}_{3}$ 
at the location. The reason for this is that we then have

$$
\begin{aligned}
E\left[\left(s_{k}-s_{l}\right)^{2}\right] & =2 \omega_{2}\left[1-\exp \left(-\Delta_{k l} / \omega_{3}\right)\right] \\
& \approx 2 \frac{\omega_{2}}{\omega_{3}} \Delta_{k l},
\end{aligned}
$$

for the first term of Eq. (28). The average correlation length for mainland Norway is around $60 \mathrm{~km}$, but can be as short as $5 \mathrm{~km}$ in some areas within the region of Fig. 4 having worst performance. In these areas, a multiplication of the result of Fig. 4 by $\sqrt{\Delta[\mathrm{km}]}$ will lead to an even more conservative value for the uncertainty. Results for separations larger than $10 \mathrm{~km}$ was not sought in this work, partly since the use of the corresponding height anomaly differences are uncommon for Norwegian surveyors. If $\Delta$ is large, one can also question the validity of the applied moving window approach in certain regions due to the variability of the statistics.

Now, for a model like Eq. (21), one can observe that $\Delta c_{k l}$ of Eq. (33) is small if the locations $k$ and $l$ are close together, or if they both are far away from the levelling network. As a result, the estimate of the uncertainty mentioned previously may be more representative rather than conservative in a range of circumstances. To investigate this more closely, the $\delta \Delta \tilde{\zeta}$ of Eq. (33) have been calculated for south of mainland Norway, yielding results that are dependent on the coordinates of both locations. So, to obtain a better understanding of the uncertainty's overall characteristics, the dimensionality of the problem is reduced. That is, each location $k$ defines the center of a circle with a chosen radius $\Delta$. Equation (33) is then calculated for 10 equiangular locations $l$ on this circle. The average $\left(\delta \Delta \tilde{\zeta}_{k l}\right)_{\text {aver }}$ of these 10 values is then computed, and represented as a fraction $\kappa$ of the square root of the conservative first term of Eq. (33)

$$
\kappa=\frac{\left(\delta \Delta \tilde{\zeta}_{k l}\right)_{\mathrm{aver}}}{\sqrt{2 \hat{\omega}_{2}\left[1-\exp \left(-\Delta / \hat{\omega}_{3}\right)\right]}} .
$$

The variation of this ratio for $\Delta=10 \mathrm{~km}$ is shown in Fig. 5, with averages at other distances given in Table 4, for the NMA2013v30 quasigeoid model.

Table 4: The averages (and minimums) of $\kappa$ of Eq. (35) for the south of mainland Norway are given below.

\begin{tabular}{ccccc}
\hline$\Delta$ & $1 \mathrm{~km}$ & $2 \mathrm{~km}$ & $5 \mathrm{~km}$ & $10 \mathrm{~km}$ \\
\hline \hline$\kappa$ & $0.98(0.82)$ & $0.97(0.72)$ & $0.92(0.61)$ & $0.86(0.55)$ \\
\hline
\end{tabular}

As can be seen, the general reduction in uncertainty is moderate for the small separations studied. However, not

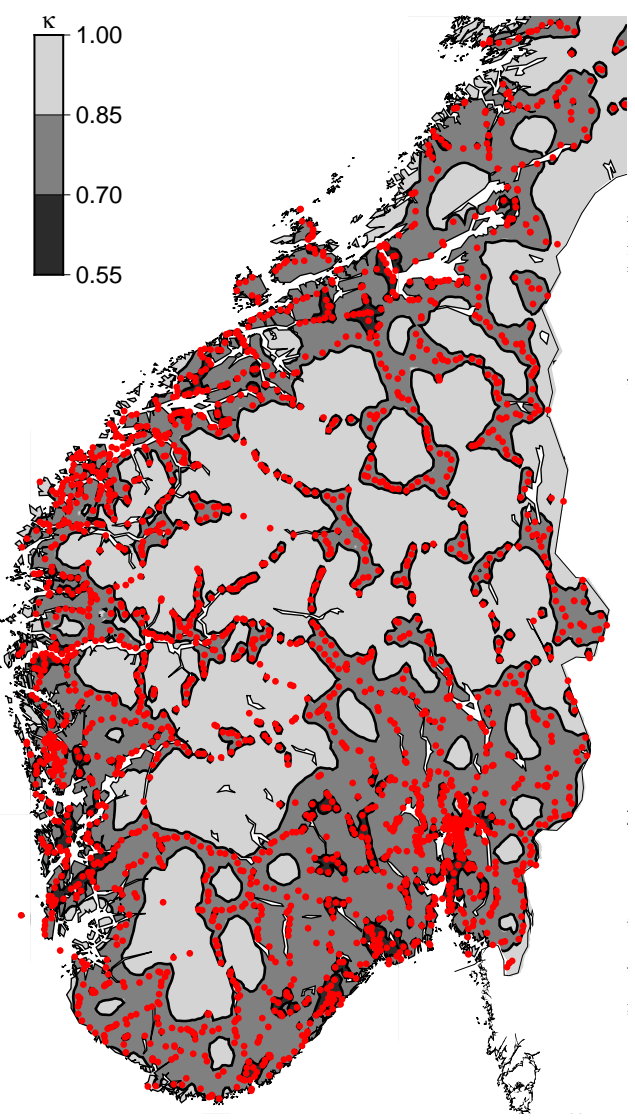

Figure 5: The relative reduction $1-\kappa$ in uncertainty due to collocation, for spatial separations of $\Delta=10 \mathrm{~km}$, can be inferred from the shown distribution of $\kappa$ given by Eq. (35). Normal heights that realize NN2000 are represented by red dots (the network).

too far from the network, the uncertainty can be reduced appreciably.

Finally, the conservative first term of Eq. (33) is recomputed for the same region of Fig. 4, but now using the gravimetric height anomalies of the NKG2015 quasigeoid model [41]. According to these results, the square root of the conservative component is reduced to below $0.9 \mathrm{~cm}$ for most of the area shown in Fig. 4, with values below $0.6 \mathrm{~cm}$ over large regions. Clearly, considerable improvements can be expected with the use of the latter gravimetric quasigeoid in the densification of future height systems. Also, the reduced variability of the signal properties is more at terms with the moving window approach of this work.

The better performance of the NKG2015 quasigeoid model may, for instance, be due to the use of better global geopotential models and more homogeneous terrestrial gravity measurements. However, highly increased consistency between the height anomalies of NN2000 and gravimetric quasigeoids developed at the Norwegian Mapping 
Authority is observed when terrain models with higher resolution are applied in the computation of the latter (colleague 0 . Omang, personal communication).

\section{Interpretation}

Throughout this work, $E(\cdot)$ has been described as the statistical operator, and the noise and signal as spatial stochastic processes. The approximate normal distribution of the prediction errors shown as Fig. 3 is, for instance, at terms with this view (Central Limit Theorem). From Fig. 2 one can also see that Eq. (23), which is embedded in a statistical framework, seems descriptive for the dispersion of the empirical variogram.

We are, however, initially free to define the nature of the signal and the content of $E(\cdot)$, but its meaning is restricted by how the a priori error covariance $R$ of Eq. (6) is determined from observations. In this work, the applied $R$ at each location is based on the implicit assumption that the empirical variogram converges locally to a continuous curve defined by Eq. (22). If this is indeed the case, the calculated uncertainty can be viewed as values towards which means of spatially distributed samples converge, independent of further interpretation.

As for the convergence of sample means towards an expectancy, this will depend not only on the sample's size, but also on the degree of correlation between its elements. In general, the rate of convergence is dependent on the nature of the signal, i.e. if it is indeed a stochastic process having a certain distribution.

This discussion emphasizes an important restriction in the given results, namely that the essential $R$ from Eq. (6) is based on the observations available. That is, the levelling network is biased towards roads and valleys. In regions not well covered by the network, the actual signal statistics, and therefore also the uncertainties, may behave differently than what has been presented.

\section{Summary}

A network of pointwise available height anomalies, derived from levelling and GPS observations, was densified by adjusting a gravimetric quasigeoid model (NMA2013v30) using least-squares collocation. The purpose of this work was to obtain a measure of uncertainty for the corresponding height anomaly differences over spatial separations less than $10 \mathrm{~km}$, motivated by how the resulting Corrector Surface Model (CSM) commonly is applied by Norwegian surveyors.
To perform the densification, and compute formal uncertainties, the discrepancy between the height anomalies of the network and the gravimetric quasigeoid needed to be modelled. In this work ${ }^{4}$, the parameters of an isotropic model were determined locally, to capture large scale inhomogeneities. For this purpose, the variogram approach to the determination of the discrepancy's magnitude and persistence with spatial separation was applied, thereby reducing the effect of modelling errors.

The resulting dispersion of prediction errors was seen to be consistent with a model that is representative. This consistency was observed locally, and over various distances at terms with how least-squares collocation is performed. These results strengthened the confidence in the formal uncertainties as representative.

A map of uncertainties related to the height anomaly differences of the gravimetric quasigeoid was then computed for spatial separations of $1 \mathrm{~km}$, showing significant large scale variations across the south of mainland Norway. It was argued that this uncertainty, in most regions, scales as $\sqrt{\Delta}$ for the small separations considered. The improvement achieved by adjusting the gravimetric quasigeoid to the network using collocation was seen to be moderate in general, but could be appreciable near the network. The relative effect of collocation was seen to improve with increasing distance.

Finally, results of a similar analysis based on the NKG2015 gravimetric quasigeoid model showed that, if applied, considerably reduced uncertainties can be expected.

Acknowledgement: The author thanks colleague Olav Vestøl for clarifications. The computations were performed with Python 3 (www.python.org), using packages NumPy and SciPy. The maps were produced with the Generic Mapping Tool [37].

\section{References}

[1] Brown N.J., McCubbine J.C., Featherstone W.E., Gowans N., Woods A. and Baran I., 2018, AUSGeoid2020 combined

4 To compute the official Norwegian Corrector Surface Model HREF, a different method is currently applied. For the computation of HREF, the gravimetric height anomalies are adjusted to the network three times using successively predefined smaller and shorter signal magnitudes and correlations lengths, respectively. The result is similar to the corresponding CSM based on local statistics. As for predictions of height anomaly differences over the fairly short spatial separations of this work, computations show that the presented uncertainties are representative also for the official HREF. 
gravimetric-geometric model: location-specific uncertainties and baseline-length-dependent error decorrelation, J. Geod., 92, 1457-1465.

[2] Cressie N., 1985, Fitting variogram models by weighted least squares, Math. Geol. 17(5), 563-586.

[3] Cressie N., 2015, Statistics for Spatial Data, Revised Edition, John Wiley \& Sons, Inc.

[4] Ekman M., 1989, Impacts of geodynamic phenomena on systems for height and gravity, Bull. Geod., 63, 281-296.

[5] Featherstone W.E., McCubbine J.C., Brown N.J., Claessens S.J., Filmer M.S. and Kirby J.F., 2018, The first Australian gravimetric quasigeoid model with location-specific uncertainty estimates, J. Geod., 92, 149-168.

[6] Fotopoulos G., Kotsakis C. and Sideris M.G., 2003, How accurately can we determine orthometric height differences from GPS and geoid data? J. Surv. Eng., 129(1), 1-10.

[7] Geary R.C., 1954, The contiguity ratio and statistical mapping, The Incorp. Stat., 5(3), 115-145.

[8] Genton M.G., 1998, Variogram fitting by generalized least squares using an explicit formula for the covariance structure, Math. Geol., 30(4), 323-345.

[9] Goldberger A.S., 1962, Best linear unbiased prediction in the generalized linear regression model, J. Am. Stat. Assoc., 57(298), 369-375.

[10] Harville D.A., 1974, Bayesian inference for variance components using only error contrasts, Biometrika, 61(2), 383-385.

[11] Harville D.A., 1977, Maximum likelihood approaches to variance component estimation and to related problems, J. Am. Stat. Assoc., 72(358), 320-338.

[12] Henderson C.R., 1975, Best linear unbiased estimation and prediction under a selection model, Biometrics, 31(2), 423-447.

[13] Huang C., Zhang H. and Robeson S.M., 2011, On the validity of commonly used covariance and variogram functions on the sphere, Math. Geosci., 43(6), 721-733.

[14] Häkli P., Lidberg M., Jivall L., Nørbech T., Tangen O., Weber M., Pihlak P., Aleksejenko I. and Paršeliunas E., 2016, The NKG2008 GPS campaign - final transformation results and a new common Nordic reference frame, J. Geod. Sci., 6, 1-33.

[15] Kearsley A.H.W., 1986, Data requirements for determining precise relative geoid heights from gravimetry, J. Geophys. Res., 91(B9), 9193-9201.

[16] Kim S.-K., Park J., Gillins D. and Dennis M., 2018, On determining orthometric heights from a corrector surface model based on levelling observations, GNSS, and a geoid model, J. App. Geod., 12(4), 323-333.

[17] Koch K.R., 1999, Parameter estimation and hypothesis testing in linear models, Second Edition, Springer-Verlag, Berlin, Heidelberg.

[18] Kotsakis C. and Sideris M.G., 1999, On the adjustment of combined GPS/levelling/geoid networks, J. Geod., 73, 412-421.

[19] Magnus J.R. and Neudecker H., 1999, Matrix differential calculus with applications in statistics and econometrics, Revised Edition, John Wiley, Chichester.

[20] Marchant B.P. and Lark R.M., 2007, Robust estimation of the variogram by residual maximum likelihood, Geoderma, 140, 62-72.

[21] Matheron G., 1963, Principles of geostatistics, Econ. Geol., 58, 1246-1266.

[22] Moritz H., 1980, Advanced physical geodesy, Wichmann, Karlsruhe.
[23] Mysen E., 2014, On the computation of reliable formal uncertainties in the densification of GPS-levelling networks by leastsquares collocation, J. Geod., 88, 917-926.

[24] Mäkinen J. and Ihde J., 2009, The permanent tide in height systems, In: Sideris, M.G. (ed.) Observing our Changing Earth. International Association of Geodesy Symposia, vol 133, 81-87, Springer, Berlin, Heidelberg.

[25] Omang O.C.D. and Forsberg R., 2000, How to handle topography in practical geoid determination: three examples, J. Geod. 74, 458-466.

[26] Patterson H.D. and Thompson R., 1971, Recovery of inter-block information when block sizes are unequal, Biometrika, 58(3), 545-554.

[27] Petit G. and Luzum B. (eds.), 2010, IERS Conventions (2010), IERS Technical Note No. 36.

[28] Poutanen M., Vermeer M. and Mäkinen J., 1996, The permanent tide in GPS positioning, J. Geod., 70, 499-504.

[29] Press W.H., Teukolsky S.A., Vetterling W.T. and Flannery B.P., 2006, Numerical Recipes in Fortran 77, The Art of Scientific Computing. Second Edition. Cambridge University Press.

[30] Schoenberg I.J., 1938, Metric spaces and positive definite functions, Trans. Amer. Math. Soc., 44, 522-536.

[31] Smith D.A. and Roman D.R., 2001, GEOID99 and G99SSS: 1-arcminute geoid models for the United States, J. Geod., 75, 469490.

[32] Strang van Hees G., 1986, Precision of the geoid, computed from terrestrial gravity measurements, Manuscripta Geodaetica, 11, 1-14.

[33] Teunissen P.J.G. and Amiri-Simkooei A.R., 2008, Least-squares variance component estimation, J. Geod., 82, 65-82.

[34] Tscherning C.C., Forsberg R. and Knudsen P., 1992, Description of the GRAVSOFT package for geoid determination, In: Holota $P$, Vermeer $M$ (eds) Proceedings of 1st continental workshop on the geoid in Europe, Research Institute of Geodesy, Topography and Cartography, Prague, 327-334.

[35] Vestøl 0., 2006, Determination of postglacial land uplift in Fennoscandia from leveling, tide-gauges and continuous GPS stations using least-squares collocation, J. Geod., 80, 248-258.

[36] Wang Y.M., Saleh J., Li X. and Roman D.R., 2012, The US gravimetric geoid of 2009 (USGG2009): model development and evaluation, J. Geod., 86, 165-180.

[37] Wessel P. and Smith W.H.F., 1998, New, improved version of the generic mapping tools released, EOS Trans. AGU, 79, 579.

[38] Williams S.D.P., 2003, The effect of coloured noise on the uncertainties of rates from geodetic time series, J. Geod., 76, 483494.

[39] Ågren J. and Svensson R., 2007, Postglacial land uplift model and system definition for the new Swedish height system RH 2000. Lantmäteriet, reports in geodesy and geographic information systems, Gävle, Sweden, p. 4

[40] Ågren J., Sjöberg L.E. and Kiamehr R., 2009, The new gravimetric quasigeoid model KTH08 over Sweden, J. App. Geod., 3, 143153.

[41] Ågren J., Strykowski G., Bilker-Koivula M., Omang O., Märdla S., Forsberg R., Ellmann A., Oja T., Liepinš I., Paršeliūnas E., Kaminskis J., Sjöberg L.E. and Valsson G., 2016, The NKG2015 gravimetric geoid model for the Nordic-Baltic region, 1st Joint Commission 2 and IGFS Meeting International Symposium on Gravity, Geoid and Height Systems, Thessaloniki, Greece. doi:10.13140/RG.2.2.20765.20969. 